\title{
BMJ Open Association between ischaemic bowel syndromes and androgen deprivation therapy in patients with prostate cancer: a retrospective cohort study
}

\author{
I-Ni Chiang, ${ }^{1,2}$ Chao-Yuan Huang, ${ }^{2}$ Yeong-Shiau Pu, ${ }^{2}$ Chao-Hsiang Chang,,${ }^{3,4}$ \\ Chih-Hsin Muo, ${ }^{5,6}$ Chi-Jung Chung, ${ }^{7,8}$ Ruey-Yun Wang, ${ }^{5}$ Tai-Horng Young ${ }^{1}$
}

To cite: Chiang I-N, Huang C-Y, Pu Y-S, et al. Association between ischaemic bowel syndromes and androgen deprivation therapy in patients with prostate cancer: a retrospective cohort study. BMJ Open 2017;7:e012950. doi:10.1136/bmjopen-2016012950

- Prepublication history for this paper is available online. To view these files please visit the journal online (http://dx.doi.org/10.1136/ bmjopen-2016-012950).

Received 16 June 2016 Revised 21 January 2017 Accepted 2 February 2017

CrossMark

For numbered affiliations see end of article.

Correspondence to Professor Ruey-Yun Wang; rywang0812@gmail.com

\section{ABSTRACT}

Objective: This study investigated the risk of ischaemic bowel syndrome (IBS) in androgen deprivation therapy (ADT) users to explore the longterm outcomes of patients with prostate cancer (PC) receiving ADT treatment.

Methods: We performed a population-based retrospective cohort study. All the clinical information of the study participants were acquired from the Longitudinal Health Insurance Database for Catastrophic IIIness Patients in Taiwan. We extracted data for all the patients newly diagnosed with prostate malignancy (ICD-9-CM 185 or C61 in ICD-10-CM) from 2000 to 2008. The patients were then divided into two groups: 7160 male ADT cohort receiving ADT and 7160 male non-ADT comparison group frequency matched by age and index year of ADT treatment of the ADT group. Cox proportional hazard regression was used to estimate the adjusted HR and $95 \%$ Cls of the IBS risk.

Results: No significant difference was noted in the overall incidence rate for IBS between the ADT and non-ADT cohorts $(0.86$ and 0.89 per 1000 personyear, respectively, $p=0.89$ ). Even after adjusting for potential risk factors, a 1.06-fold risk of IBS $(95 \% \mathrm{Cl}$ 0.62 to $1.82, p=0.82$ ) was observed in the ADT cohort relative to the non-ADT cohorts. Moreover, we stratified the ADT cohort by time point of ADT treatment after $P C$ diagnosis. Different IBS incidence rates were observed among the early ADT, late-ADT and non-ADT users at $0.77,1.23$ and 0.89 per 1000 person-years, respectively; nonetheless, the difference was not statistically significant. Moreover, no difference was found between the ADT treatment types and IBS risk, including sole orchiectomy, sole luteinising-hormonereleasing hormone and both.

Conclusions: Results showed that ADT treatment in patients with PC is not an independent factor for IBS incidence. Large sample sizes for patients with IBS with patients with PC who had received ADT treatment are needed for further study.

\section{INTRODUCTION}

Although Asian populations record a relatively low incidence and mortality rate in

\section{Strengths and limitations of this study}

- Nationwide population and complete medical history were analysed using claims data from the Taiwan National Health Institutes.

- Certain lifestyle-related data, such as smoking status or cancer stage and grade, were not available and not adjusted in the analysed model.

- Small numbers of ischaemic bowel syndrome incidences resulted in a wide $\mathrm{Cl}$ for the HRs.

prostate cancer $(\mathrm{PC})$, this cancer type has become highly common among male cancers. This trend may be related to the enhanced detection and westernisation. ${ }^{1}$ Androgen deprivation therapy (ADT) is a reasonable treatment option for patients with high-risk disease, very-high-risk localised disease and metastatic disease. ${ }^{2}$ ADT options mainly include operative castration with orchiectomy and chemical castration with luteinising-hormone-releasing hormone (LHRH) agonists. ${ }^{3}$ ADT has been reported to be associated with adverse effects on bone, old age, cognitive health and body composition. ${ }^{4}$

Ischaemic bowel syndrome (IBS) is a heterogeneous disorder that represents ischaemic damage to different portions of the bowel; the disease is associated with variable clinical symptoms and outcomes. ${ }^{5}$ IBS may be caused by impaired blood perfusion to the small or large bowel, including acute arterial mesenteric ischaemia, acute venous mesenteric ischaemia, non-occlusive mesenteric ischaemia, ischaemia/reperfusion injury and ischaemic colitis. ${ }^{7}$ The risk factors of IBS include old age, thromboembolic events, arrhythmia, low ejection fraction, congestive heart failure, asthma, chronic obstructive pulmonary disease (COPD), cardiomyopathy, recent myocardial infarction, ventricular aneurysm, aortic insufficiency and renal and 
Table 1 Distribution of baseline characteristics between patients with prostate malignancy with and without ADT treatment

\begin{tabular}{|c|c|c|c|c|c|}
\hline & \multicolumn{2}{|c|}{$\begin{array}{l}\text { ADT } \\
\mathrm{N}=7160\end{array}$} & \multicolumn{2}{|c|}{$\begin{array}{l}\text { Non-ADT } \\
\mathrm{N}=7160\end{array}$} & \multirow[b]{2}{*}{ p Value } \\
\hline & $\mathbf{N}$ & Per cent & $\mathbf{N}$ & Per cent & \\
\hline Age, year & & & & & 0.99 \\
\hline$<65$ & 932 & 13.0 & 932 & 13.0 & \\
\hline $65-69$ & 1008 & 14.1 & 1008 & 14.1 & \\
\hline $70-74$ & 1659 & 23.2 & 1659 & 23.2 & \\
\hline $75-79$ & 1849 & 25.8 & 1849 & 25.8 & \\
\hline $80+$ & 1712 & 23.9 & 1712 & 23.9 & \\
\hline Mean (SD) & 74.2 & $(8.16)$ & 74.2 & $(8.11)$ & 0.95 \\
\hline \multicolumn{6}{|l|}{ Comorbidity } \\
\hline CAD & 2674 & 37.4 & 3085 & 43.1 & $<0.0001$ \\
\hline Diabetes & 1771 & 24.7 & 1913 & 26.7 & 0.007 \\
\hline Stroke & 1822 & 25.5 & 2031 & 28.4 & $<0.0001$ \\
\hline Hypertension & 4698 & 65.6 & 4884 & 68.2 & 0.001 \\
\hline Hyperlipidaemia & 2148 & 30.0 & 2132 & 29.8 & 0.77 \\
\hline Lower leg fracture or surgery & 451 & 6.30 & 377 & 5.27 & 0.008 \\
\hline Asthma & 985 & 13.8 & 1006 & 14.1 & 0.61 \\
\hline COPD & 3638 & 50.8 & 4028 & 56.3 & $<0.0001$ \\
\hline \multicolumn{6}{|l|}{ Treatment } \\
\hline Prostatectomy & 3 & 0.04 & 1 & 0.01 & 0.32 \\
\hline Radiotherapy & 2905 & 40.6 & 2637 & 36.8 & $<0.0001$ \\
\hline Antiandrogens & 6743 & 94.2 & 5513 & 77.0 & $<0.0001$ \\
\hline $\begin{array}{l}\text { Duration from prostate cancer diagnosed } \\
\text { to receiving } A D T \text {, year (SD) }\end{array}$ & 0.76 & (1.51) & 0.73 & $(0.64)$ & 0.07 \\
\hline
\end{tabular}

hepatic diseases, as well as certain medications, such as bevazirumab. ${ }^{8-11}$

Previous studies showed a correlation between metabolic syndrome, diabetes, cardiovascular events and ADT. $^{12}{ }^{13}$ O'Farrell et $a l^{14}$ reported that ADT would increase the risk of thromboembolic disease. Considering that IBS is common in men with cardiovascular and thromboembolic problems, we evaluated whether IBS is also related to ADT. Several studies have addressed the relationship between ADT and subsequent risk of IBS. Thus, we conducted a population-based retrospective cohort study to explore whether ADT treatment in patients with PC would increase the risk of IBS.

\section{MATERIALS AND METHODS}

\section{Data source}

The Longitudinal Health Insurance Database for Catastrophic Illness Patient was used in this retrospective cohort study. LHID-CIP was set up by Taiwan National Health Insurance (NHI) Administration Ministry of Health and Welfare and maintained by the National Health Research Institute. This database included all medical records for each catastrophic illness patient from 1996 to 2011. In Taiwan, patients would apply a catastrophic illness card to escape the copayment of inpatient or outpatient care. These patients must acquire a physician's certificate for catastrophic illness according to the Ministry of Health and Welfare guideline. On the basis of the Personal Information Protection Act, patient identification was recorded by NHI.

\section{Study participants}

We recruited 24464 male patients newly diagnosed with PC by reviewing their medical records and using the code of International Classification of Diseases, Ninth Revision, Clinical Modification (ICD-9-CM), 185 or C61 in ICD-10-CM during 2000-2008 in LHID-CIP database. Patients with the following criteria were excluded: (1) with ADT before the date for PC diagnosis; (2) other cancer history (ICD-9-CM 140-184 and 186-208); (3) with IBS history (IBS, ICD-9-CM 557); (4) with age $<20$ years old and (5) with duration between ADT and IBS< 1 year. ADT included bilateral simple orchiectomy and administration of LHRH agonists containing leuprorelin (ATC code L02AE02), goserelin (L02AE03) and triptorelin (L02AE04).

Patients with PC were stratified into two groups on the basis of receipt of ADT. The non-ADT group was frequency matched by age strum (each 5-year strum, eg, 20-24, 25-29, 30-34 and so on) and by the index year of ADT treatment of the ADT group.

\section{Endpoint and comorbidity}

All the study subjects were followed up from the index year of ADT treatment to IBS development. The subjects that did not develop IBS were followed up until the date 


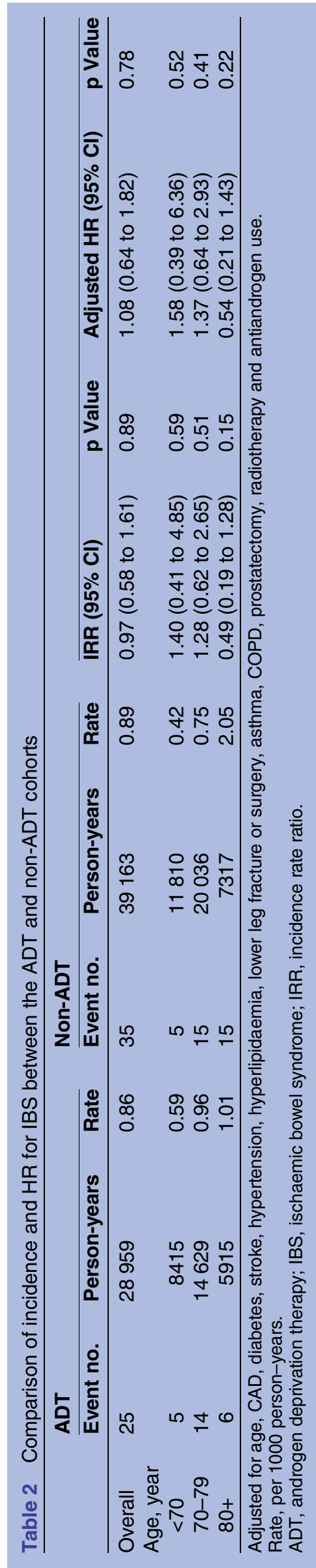

of withdrawal from the NHI programme or the end of 2011, whichever came first. Baseline comorbidity included coronary artery disease (CAD; ICD-9-CM 410414), cardiovascular disease (CVD; ICD-9-CM 430-438), diabetes mellitus (ICD-9-CM 250), hypertension (ICD-9-CM 401-405), hyperlipidaemia (ICD-9-CM 272), lower leg fracture or surgery (ICD-9-CM 820, 821 and 823 and operation code 81.51-81.54), asthma (ICD-9-CM code 493) and COPD (ICD-9-CM 491, 492 and 496). During analysis, we also considered different treatments, such as prostatectomy (operation code 79403B, 79410B, 79404B and 79405B); radiotherapy (36009B and 36012B) and antiandrogen therapy, such as bicalutamide (ATC code L02BB03), flutamide (ATC code L02BB01) and cyproterone (ATC code G03HA01) treatments.

\section{Statistical analysis}

All statistical analyses were performed using SAS software V.9.4 (SAS Institute, Cary, North Carolina, USA). The significance level was set at $\mathrm{p}<0.05$ by using two-tailed testing. We used $\chi^{2}$ test to observe the difference in age distribution $(<65,65-69,70-74,75-79$ and $80+$ years old) and the baseline comorbidity between the ADT and non-ADT groups. The differences in age and duration (from PC diagnosis and ADT treatment) between the $\mathrm{ADT}$ and non-ADT groups were evaluated using the t-test. The incidence density of IBS (per 1000 personyears) in the two groups was calculated. Poisson regression was used to assess the incidence rate ratio (IRR) in the ADT group compared with that of the non-ADT group. Cox proportional hazard regression was used to estimate the adjusted HR and 95\% CIs of IBS after adjustment for age and all baseline comorbidities. We also estimated the association between IBS and the time point for ADT treatment (early and late treatments). Patients with ADT treatment within 180 days after PC diagnosis were defined as the early group, and the patients given ADT treatment for more than 180 days after PC diagnosis were defined as the late group. The association between IBS and different ADT treatment stages (early/late) was also assessed.

\section{RESULT}

We selected 7160 patients with PC with ADT treatment and 7160 patients without ADT treatment. The mean ages in the ADT and non-ADT cohorts were 74 years (table 1). No significant difference in age and duration (from PC diagnosis and ADT treatment) was observed for the two groups. Compared with the non-ADT group, the ADT group was likely to present a low baseline comorbidity, including CAD $(37.4 \%$ vs $43.1 \%)$, diabetes (24.7\% vs $26.7 \%)$, stroke (25.5\% vs $28.4 \%)$, hypertension $(65.6 \%$ vs $68.2 \%)$ and COPD $(50.8 \%$ vs $56.3 \%)$. However, the ADT cohort achieved a higher prevalence of lower leg fracture or surgery $(6.30 \%$ vs $5.27 \%)$, as well as radiotherapy ( $40.6 \%$ vs $36.8 \%)$ and antiandrogen therapy (94.2\% vs $77 \%)$, than the non-ADT cohort. 
Table 3 Incidence and HRs for IBS in the patients who underwent different durations between the onset of prostate malignancy and ADT treatment relative to those of the non-ADT cohort

\begin{tabular}{|c|c|c|c|c|c|c|c|c|}
\hline & \multirow[b]{2}{*}{$\overline{\mathbf{N}}$} & \multirow[b]{2}{*}{ Event no. } & \multirow[b]{2}{*}{ Person-years } & \multirow[b]{2}{*}{ Rate } & \multicolumn{3}{|l|}{$\mathrm{HR}(95 \% \mathrm{Cl})$} & \multirow[b]{2}{*}{ p Value } \\
\hline & & & & & Crude & p Value & Adjusted & \\
\hline $\begin{array}{l}\text { Non-ADT } \\
\text { ADT }\end{array}$ & 7160 & 35 & 39163 & 0.89 & 1.00 & & 1.00 & \\
\hline Early & 5351 & 18 & 23266 & 0.77 & 0.87 (0.49 to 1.53$)$ & 0.62 & 0.95 (0.53 to 1.70$)$ & 0.85 \\
\hline Late & 1809 & 7 & 5692 & 1.23 & 1.38 (0.61 to 3.10$)$ & 0.44 & 1.59 (0.69 to 3.66$)$ & 0.28 \\
\hline
\end{tabular}

Adjusted for age, CAD, diabetes, stroke, hypertension, hyperlipidaemia, lower leg fracture or surgery, asthma, COPD, prostatectomy, radiotherapy and antiandrogen use.

Early: duration between the date of onset of prostate cancer and ADT treatment $\leq 180$ days.

Late: duration between the date of onset of prostate cancer and ADT treatment $>180$ days.

Rate, per 1000 person-years.

ADT, androgen deprivation therapy; IBS, ischaemic bowel syndrome.

During follow-up from 2000 to 2011, 25 and 35 patients developed IBS in the ADT (28959 personyears) and non-ADT cohorts (39 163 person-years) (table 2). For the ADT and non-ADT cohorts, the average follow-up years of ADT treatment and IBS incidence were 3.48 (incident rate: 0.86 per 1000 personyears) and 4.24 years (incident rate: 0.89 per 1000 person-years), respectively. Compared with the non-ADT cohort, the IBS risk in the ADT cohort was 0.97 (95\% CI 0.58 to 1.61 ) and 1.06 (95\% CI 0.62 to 1.82 ) in the crude and adjusted Cox model, respectively. Regardless of age group, no significant difference in IBS incidence was observed between the two cohorts.

Table 3 presents the association between IBS and the time point for ADT treatment. The patients with late ADT treatment show a high IBS incidence than that of the non-ADT cohort (1.23 vs 0.89 per 1000 personyears) but the difference was not statistically significant.

The association between IBS and the different types of ADT treatment is shown in table 4. The IBS incidence values were $0.89,0.92,0.92$ and 0.35 per 1000 person-years in the patients without ADT treatment, with orchiectomy, with LHRH agonist therapy and with both treatments, respectively. The patients who underwent orchiectomy or LHRH treatment showed higher IBS incidence, but the difference did not reach statistical significance.

\section{DISCUSSION}

ADT could be beneficial to symptomatic advanced PC. The treatment may also serve as a neoadjuvant therapy in patients with PC receiving radiotherapy and may be efficacious for metastatic disease; however, ADT is associated with certain adverse effects and complications. ${ }^{15}$ These adverse effects include decreased bone mineral density, osteoporosis, weight gain, reduced muscle mass, aggravated insulin resistance, decreased libido, reduced sexual function, hot flushes, gynaecomastia, reduced testicular size, anaemia, depression, cognitive decline and fatigue. ${ }^{4}$ Chung et al reported a significant relationship between ADT with gonadotropin-releasing hormone $(\mathrm{GnRH})$ agonists and an increased pneumonia risk. ${ }^{3}$ $\mathrm{ADT}$ is a well-established treatment for advanced and metastatic PC and is mainly administered with bilateral simple orchiectomy (surgical castration) or LHRH therapy (medical castration). ${ }^{16}$ Oral antiandrogen is not usually used as monotherapy. We evaluated the medication history from our records and found that $94.2 \%$ and $77 \%$ of the patients with PC who received and did not receive $\mathrm{ADT}$, respectively, also received antiandrogens. Thus, we mainly focused on LHRH in our study. Among 24464 patients with PC, only $257(1.05 \%)$ patients used LHRH agonists as single treatment. Therefore, we could not eliminate the patients treated with combined antiandrogens. Seaman et al compared the risk of venous thromboembolism among patients treated with different antiandrogens and LHRH agonists. The group found that venous thromboembolism was associated only with cyproterone acetate, whereas the other antiandrogens did not contribute to thromboembolic event. ${ }^{17}$ In our series, ADT users presented a significantly higher percentage of lower leg fracture/surgery than the non-ADT

Table 4 Incidence and HRs for IBS under different ADT treatments relative to those in non-ADT patients

\begin{tabular}{|c|c|c|c|c|c|c|c|c|}
\hline & \multirow[b]{2}{*}{$N$} & \multirow[b]{2}{*}{ Event no. } & \multirow[b]{2}{*}{ Person-years } & \multirow[b]{2}{*}{ Rate } & \multicolumn{3}{|l|}{ HR (95\% Cl) } & \multirow[b]{2}{*}{ p-value } \\
\hline & & & & & Crude & p-value & Adjusted & \\
\hline Non-ADT & 7160 & 35 & 39163 & 0.89 & 1.00 & & 1.00 & \\
\hline \multicolumn{9}{|l|}{ ADT treatment } \\
\hline Orchiectomy & 1754 & 7 & 7574 & 0.92 & 1.03 (0.46 to 2.33$)$ & 0.94 & $1.03(0.45$ to 2.35$)$ & 0.94 \\
\hline $\mathrm{LHRH}$ & 4710 & 17 & 18568 & 0.92 & $1.02(0.57$ to 1.83$)$ & 0.93 & $1.17(0.64$ to 2.15$)$ & 0.60 \\
\hline Both & 696 & 1 & 2817 & 0.35 & 0.40 (0.05 to 2.90$)$ & 0.36 & 0.45 (0.06 to 3.30$)$ & 0.43 \\
\hline
\end{tabular}

Adjusted for age, CAD, diabetes, stroke, hypertension, hyperlipidaemia, lower leg fracture or surgery, asthma, COPD, prostatectomy, radiotherapy and antiandrogen use.

$\mathrm{ADT}$, androgen deprivation therapy; IBS, ischaemic bowel syndrome; LHRH, luteinising-hormone-releasing hormone. 
users $(6.30 \%$ vs $5.27 \%, \mathrm{p}<0.01)$. This result is identical to that in previous literature. ${ }^{412} 15$

The cardiovascular effects of ADT have been reported by several studies, but the results are controversial. ${ }^{12}$ Wall $e t a l^{18}$ observed that prolonged ADT is associated with accumulating CVD risk factors, such as reduced cardiorespiratory capacity and decreased resting metabolic rate. Short-term (3-6 months) ADT results in the development of hyperinsulinaemia, and long-term ADT ( $\geq 12$ months) yields a higher prevalence of diabetes and metabolic syndrome. ${ }^{19}$ In men, testosterone is inversely correlated with the level of low-density lipoprotein cholesterol and positively correlated with the level of highdensity lipoprotein (HDL) cholesterol. ${ }^{20}$ Meanwhile, ADT is known to increase HDL and total cholesterol in patients with $\mathrm{PC} .{ }^{21}$ This aspect means that androgen suppression with ADT variably influences the HDL-testosterone correlation relative to those of non-diabetic men. Several studies also supported that ADT is associated with thromboembolic cardiovascular events, including aortic atherosclerosis, $\mathrm{CAD}$, myocardial infarction and sudden cardiac death. ${ }^{22-24}$ However, the studies where ADT exerted significantly negative cardiovascular effects mainly involved Caucasian population. ${ }^{25} \mathrm{~A}$ recent study in Asia showed no increase in risk of coronary heart disease in the Chinese/Taiwanese patients with PC who received ADT. ${ }^{26}$ The findings are supported by several other studies. ${ }^{13}{ }^{27}$ In the present study, we considered the abovementioned ADT-related risk factors in the models. However, ADT did not significantly increase IBS risk. In the present study, the mean age of the patients with PC was 74 years old, which is older than those in other countries, including Japan and in the Caucasian population. ${ }^{28}$ Late age PC diagnosis in Taiwan could be blinding the ADT effects because the risks of CVD, stroke and diabetes increase with age.

IBS is an abdominal emergency caused by impaired intestinal blood perfusion. ${ }^{7}$ At present, mesenteric ischaemia presents a high mortality rate, ranging between $50 \%$ and $90 \% .{ }^{29}$ Thus, prompt diagnosis and risk factor identification are crucial for IBS management. The main predisposing factors for IBS are vascular factors and bowel factors. Hypoperfusion, vascular surgery requiring aortic clamping, vasospasm, vasoconstrictor drugs and thromboembolism from hypercoagulable states or cardiac emboli may lead to IBS. ${ }^{30}$ O'Farrell et al assessed the risk of thromboembolic disease in patients with PC undergoing ADT. The group noted that the incidence of deep vein thrombosis and pulmonary embolism increases with prolonged ADT usage and recommended that only men with relevant indication should receive systemic ADT. ${ }^{14}$ The study also showed an increased thrombotic risk in the patients who switched from antiandrogen therapy to GnRH agonist therapy, although these agonists were not considered in our present analysis. ${ }^{14}$ Teoh $e t a \hat{l}^{1}$ observed that ADT users presented an increased risk of ischaemic stroke relative to non-ADT users in patients with PC. Old age
(HR 1.13), hyperlipidaemia (HR 4.61) and ADT (HR $3.32)$ were associated with ischaemic stroke. Ultee $e t a l^{32}$ also reported an increased risk for bowel ischaemia in smokers, leading to COPD. ${ }^{11}$ Longstreth et al found that acute large bowel ischaemia is independently associated with COPD (adjusted OR 3.13). ${ }^{10}$ Thus, we considered the COPD variable in our adjusted models. Considering that ADT is reportedly associated with diabetes, dyslipidaemia and CVD, which are all related to thromboembolic events, we determined whether the ADT users presented a higher risk of IBS development. In general, patients with PC with good clinical conditions received ADT treatment. Therefore, the ADT group in the present study exhibited a lower prevalence of $\mathrm{CAD}$, diabetes, stroke, hypertension and COPD. We then considered these factors in the multivariate regression models. Accordingly, no difference in IBS incidence was observed between the ADT and non-ADT users. Although the late-ADT users presented higher HRs of IBS compared with the early ADT and non-ADT users, a significant difference was not reached.

Although the data analysed in the study were obtained from the nationwide-based LHID-CIP, several limitations must be considered. First, the follow-up duration of study cohort was $\sim 10$ years (2000-2011). Considering the medical and medication history, as well as frequency matching in the study design, we limited the ADT and non-ADT groups and IBS incidence to a small sample size in the present study. This aspect resulted in the wide CI for risk. Second, all the patients with PC in our study possessed an average age of 74 years and exhibited high mortality during follow-up (48.2\% in the ADT group and $35.6 \%$ in the non-ADT group). Yu et $a l^{33}$ reported that two genetic markers, namely, AKR1C3 rs12529 and AR-CAG repeat length, are significantly associated with PC-specific mortality. In addition, the genetic polymorphism of AKR1C3 rs12529 could also minimise ADT-related impact on quality of life in patients with PC. ${ }^{34}$ The effect of the genetic variability on IBS risk was not considered in the present analysis. However, we further examined and found no violation for the assumption of the Cox proportional hazard regression $(p=0.87)$. Finally, several important clinical variables, such as prostate serum antigen levels, cancer stage and grade and smoking habits, were not acquired from the LHID-CIP. For cigarette smoking, we attempted to use the COPD variables as proxy indicators for tobacco smoking and further adjusted the variable in the multivariable models. However, the association between ADT therapy and IBS risk remains statistically insignificant.

\section{CONCLUSION}

No significant difference in HR for IBS was observed between ADT-treated and non-ADT-treated patients with PC. Large-scale studies of IBS events and other related clinical variables must be conducted to determine the association between ADT and IBS risk. 
Author affiliations

${ }^{1}$ Institute of Biomedical Engineering, College of Medicine and College of Engineering, National Taiwan University, Taipei, Taiwan

${ }^{2}$ Department of Urology, National Taiwan University Hospital, College of Medicine, National Taiwan University, Taipei, Taiwan

${ }^{3}$ Department of Urology, China Medical University and Hospital, Taichung, Taiwan

${ }^{4}$ Department of Medicine, College of Medicine, China Medical University and Hospital, Taichung, Taiwan

${ }^{5}$ Department of Public Health, China Medical University, Taichung, Taiwan

${ }^{6}$ Management Office for Health Data, China Medical University and Hospital,

Taichung, Taiwan

${ }^{7}$ Department of Health Risk Management, College of Public Health, China Medical University, Taichung, Taiwan

${ }^{8}$ Department of Medical Research, China Medical University and Hospital, Taichung, Taiwan

Contributors C-JC, I-NC, C-YH, Y-SP, C-HC, R-YW and T-HY partly contributed to the conception and design of the work; C-JC, R-YW and C-HM contributed to the analysis of the data and wrote the manuscript; C-HM performed the data analysis. All authors have read the manuscript and approved the final version for submission to BMJ Open and those they accept responsibility for the manuscript's contents.

Funding This study is supported in part by the Taiwan Ministry of Health and Welfare Clinical Trial and Research Center of Excellence

(MOHW105-TDU-B-212-133019); China Medical University Hospital;

Academia Sinica Taiwan Biobank Stroke Biosignature Project

(BM10501010037); Tseng-Lien Lin Foundation, Taichung, Taiwan; Taiwan

Brain Disease Foundation, Taipei, Taiwan and Katsuzo and Kiyo Aoshima

Memorial Funds, Japan.

Competing interests All authors have disclosed any potential competing financial interests regarding the submitted article.

Ethics approval This study was approved by the Research Ethics Committee of China Medical University and hospital (CMUH104-REC2-115).

Provenance and peer review Not commissioned; externally peer reviewed.

Data sharing statement No additional data are available.

Open Access This is an Open Access article distributed in accordance with the Creative Commons Attribution Non Commercial (CC BY-NC 4.0) license, which permits others to distribute, remix, adapt, build upon this work noncommercially, and license their derivative works on different terms, provided the original work is properly cited and the use is non-commercial. See: http:// creativecommons.org/licenses/by-nc/4.0/

\section{REFERENCES}

1. Pu YS, Chiang HS, Lin CC, et al. Changing trends of prostate cancer in Asia. Aging Male 2004;7:120-32.

2. Mohler JL, Armstrong AJ, Bahnson RR, et al. Prostate cancer, version 1.2016. J Natl Compr Canc Netw 2016;14:19-30.

3. Hsieh RL, Huang YL, Shiue HS, et al. Arsenic methylation capacity and developmental delay in preschool children in Taiwan. Int $J$ Hyg Environ Health 2014;217:678-86.

4. Nguyen PL, Alibhai SM, Basaria S, et al. Adverse effects of androgen deprivation therapy and strategies to mitigate them. Eur Urol 2015;67:825-36.

5. Greenwald DA, Brandt LJ, Reinus JF. Ischemic bowel disease in the elderly. Gastroenterol Clin North Am 2001;30:445-73.

6. Reinus JF, Brandt LJ, Boley SJ. Ischemic diseases of the bowel. Gastroenterol Clin North Am 1990;19:319-43.

7. Reginelli A, lacobellis F, Berritto D, et al. Mesenteric ischemia: the importance of differential diagnosis for the surgeon. BMC Surg 2013;13(Suppl 2):S51.

8. Wiesner W, Khurana $\mathrm{B}$, Ji H, et al. CT of acute bowel ischemia. Radiology 2003;226:635-50.

9. Lordick F, Geinitz H, Theisen J, et al. Increased risk of ischemic bowel complications during treatment with bevacizumab after pelvic irradiation: report of three cases. Int J Radiat Oncol Biol Phys 2006;64:1295-8.
10. Longstreth GF, Yao JF. Diseases and drugs that increase risk of acute large bowel ischemia. Clin Gastroenterol Hepatol 2010;8:49-54

11. Ogawa K, Kishi K. [Etiological and exacerbation factors for COPD. Air pollution]. Nihon Rinsho 2016;74:743-6.

12. Rhee $\mathrm{H}$, Gunter $\mathrm{JH}$, Heathcote $\mathrm{P}$, et al. Adverse effects of androgen-deprivation therapy in prostate cancer and their management. BJU Int 2015;115(Suppl 5):3-13.

13. Morgans AK, Fan KH, Koyama $\mathrm{T}$, et al. Influence of age on incident diabetes and cardiovascular disease in prostate cancer survivors receiving androgen deprivation therapy. J Urol 2015; 193:1226-31.

14. O'Farrell S, Sandström K, Garmo H, et al. Risk of thromboembolic disease in men with prostate cancer undergoing androgen deprivation. BJU Int 2016;118:391-8.

15. Heidenreich A, Bastian PJ, Bellmunt J, et al. EAU guidelines on prostate cancer. Part II: Treatment of advanced, relapsing, and castration-resistant prostate cancer. Eur Urol 2014;65:467-79.

16. Lin $\mathrm{YH}, \mathrm{Chen} \mathrm{CL}$, Hou CP, et al. A comparison of androgen deprivation therapy versus surgical castration for patients with advanced prostatic carcinoma. Acta Pharmacol Sin 2011;32:537-42.

17. Seaman HE, Langley SE, Farmer RD, et al. Venous thromboembolism and cyproterone acetate in men with prostate cancer: a study using the General Practice Research Database. BJU Int 2007;99:1398-403.

18. Wall BA, Galvão DA, Fatehee N, et al. Reduced cardiovascular capacity and resting metabolic rate in men with prostate cancer undergoing androgen deprivation: a comprehensive cross-sectional investigation. Adv Urol 2015;2015:976235.

19. Basaria S. Androgen deprivation therapy, insulin resistance, and cardiovascular mortality: an inconvenient truth. J Androl 2008;29:534-9.

20. Haffner SM, Mykkanen L, Valdez RA, et al. Relationship of sex hormones to lipids and lipoproteins in nondiabetic men. $J$ Clin Endocrinol Metab 1993;77:1610-15.

21. Smith MR, Finkelstein JS, McGovern FJ, et al. Changes in body composition during androgen deprivation therapy for prostate cancer. J Clin Endocrinol Metab 2002;87:599-603.

22. Choong K, Basaria S. Emerging cardiometabolic complications of androgen deprivation therapy. Aging Male 2010;13:1-9.

23. Keating NL, O'Malley AJ, Smith MR. Diabetes and cardiovascular disease during androgen deprivation therapy for prostate cancer. $J$ Clin Oncol 2006;24:4448-56.

24. Jones RD, Malkin CJ, Channer KS, et al. Low levels of endogenous androgens increase the risk of atherosclerosis in elderly men: further supportive data. J Clin Endocrinol Metab 2003;88:1403-4; author reply 4.

25. Nguyen PL, Je Y, Schutz FA, et al. Association of androgen deprivation therapy with cardiovascular death in patients with prostate cancer: a meta-analysis of randomized trials. JAMA 2011;306:2359-66.

26. Wang W, Xu L. [The clinical features and risk factors for 89 cases of ischemic colitis]. Zhonghua Nei Ke Za Zhi 2012:51:769-73.

27. Bolla $M$, Van Tienhoven $G$, Warde $P$, et al. External irradiation with or without long-term androgen suppression for prostate cancer with high metastatic risk: 10-year results of an EORTC randomised study. Lancet Oncol 2010;11:1066-73.

28. Chen $\mathrm{CH}$, Tzai TS, Huang SP, et al. Clinical outcome of Taiwanese men with metastatic prostate cancer compared with other ethnic groups. Urology 2008;72:1287-92.

29. Elder K, Lashner BA, AI Solaiman F. Clinical approach to colonic ischemia. Cleve Clin J Med 2009;76:401-9.

30. Sherid M, Sifuentes H, Samo S, et al. Ischemic colitis: a forgotten entity. Results of a retrospective study in 118 patients. J Dig Dis 2014;15:606-13.

31. Teoh JY, Chiu PK, Chan SY, et al. Risk of ischemic stroke after androgen deprivation therapy for prostate cancer in the Chinese population living in Hong Kong. Jpn J Clin Oncol 2015;45:483-7.

32. Ultee $\mathrm{KH}$, Zettervall SL, Soden PA, et al. Incidence of and risk factors for bowel ischemia after abdominal aortic aneurysm repair. $J$ Vasc Surg 2016;64:1384-91.

33. Yu CC, Huang SP, Lee YC, et al. Molecular markers in sex hormone pathway genes associated with the efficacy of androgen-deprivation therapy for prostate cancer. PLoS ONE 2013;8:e54627.

34. Karunasinghe N, Zhu Y, Han DY, et al. Quality of life effects of androgen deprivation therapy in a prostate cancer cohort in New Zealand: can we minimize effects using a stratification based on the aldo-keto reductase family 1 , member C3 rs12529 gene polymorphism? BMC Urol 2016;16:48 